\title{
The Galactic Halo and CDM
}

\author{
Michael R. Merrifield \\ School of Physics \& Astronomy, University of Nottingham, University \\ Park, Nottingham, NG7 2RD, UK
}

\begin{abstract}
This paper reviews the available information on the central density distribution and shape of the Milky Way's halo. At present, there is no strong evidence that the Milky Way's halo properties conflict with the predictions of cold dark matter (CDM): a primordial central power law cusp can be accommodated by the observations, and the current constraints on flattening are also consistent with the predictions of the theory. If you want to pick a fight with CDM, then the Milky Way is probably not the place to do it.
\end{abstract}

\section{Introduction}

The cosmological principle states that there are no special places in the Universe. A simple corollary of this principle is that we cannot be anywhere special in the Universe, and this assumption has been supported by a long line of discoveries running all the way from Copernicus to Shapley demonstrating that we live in the sprawling suburbs of the Milky Way. It also, of course, means that the Milky Way is a very ordinary spiral galaxy. Although we should not be fooled into thinking that all galaxies are necessarily like the Milky Way, we have a very useful test of any theory of galaxy formation in that properties of the Milky Way should lie within the range of what is considered "normal" by the theory.

In some ways, we are making life hard for ourselves by using the Milky Way for such tests, since many observations that are fairly trivial in external galaxies are rather challenging in our own Galaxy. Our position within the Milky Way complicates the issue for several reasons. First, the geometry is more complex than for distant systems, since there is no simple relationship between angular and linear scale. Second, since parts of the Milky Way lie in all directions, large surveys are generally required to study its properties. Third, living right in the dust lane of the Galaxy makes obscuration more of a factor than in most external systems. However, these difficulties mainly affect attempts to measure global properties of the Milky Way, such as its overall morphology. For smaller-scale phenomena, our proximity to the action makes the Galaxy an ideal laboratory.

In particular, the Milky Way offers the unique possibility for an in situ local measurement of halo properties that is not attainable for any other system. By measuring local stellar kinematics, Kuijken \& Gilmore (1991) obtained a fairly robust estimate for the total amount of mass in the solar neighbourhood within $1.1 \mathrm{kpc}$ of the Galactic plane, $\Sigma_{1.1} \approx 70 M_{\odot} \mathrm{pc}^{-2}$. Much of this mass can be attributed to the visible components of the Galaxy: $\sim 25 M_{\odot} \mathrm{pc}^{-2}$ is contributed 
by the normal stellar component, and $\sim 15 M_{\odot} \mathrm{pc}^{-2}$ comes from the interstellar medium (Olling \& Merrifield 2001). However, this still leaves around $30 M_{\odot} \mathrm{pc}^{-2}$ unaccounted for, which presumably must be attributed to the dark matter halo. If the scaleheight of this dark component is large compared to the $1.1 \mathrm{kpc}$ within which the mass is measured, then we can obtain a direct measure of the local dark matter density, $\rho^{\mathrm{DM}}\left(R_{0}, 0\right) \approx 0.014 M_{\odot} \mathrm{pc}^{-3}$. Here, we have made explicit the Galactocentric cylindrical polar coordinates which locate the Sun at a radius $R_{0} \approx 8.5 \mathrm{kpc}$ from the Galactic center (Kerr \& Lynden-Bell 1986), approximately in the $z=0$ plane. As we shall see below, this single data point is probably the most important contribution that the Milky Way can make to the general study of dark matter, since it offers a unique localized measurement that we cannot make at any other point, or in any other dark matter halo.

\section{Central Cusp}

One immediate constraint that the local dark matter measurement offers is on the power law slope of any central cusp in the dark matter distribution. Almost all simulations indicate that cold dark matter (CDM) should form halos with a density distribution that goes from a relatively shallow power law at small radii to a steeper one at large radii, with a transition occuring at a radius $r_{s}$ that depends on the mass of the halo. For an object the size of the Milky Way, this transition should occur at $r_{s} \sim 20 \mathrm{kpc}$, well outside $R_{0}$, so the density distribution in the inner Galaxy should follow a single power law rather closely.

If we write this power law as $\rho(r) \propto r^{-\alpha}$ (where we have assumed, for the moment, that the halo is spherical), and normalize the distribution using $\rho^{\mathrm{DM}}\left(R_{0}, 0\right)$, we find that the total mass of dark matter interior to the Solar radius is

$$
M^{\mathrm{DM}}\left(R_{0}\right)=\frac{4 \pi}{3-\alpha} \rho^{\mathrm{DM}}\left(R_{0}, 0\right) \times R_{0}^{3} \approx \frac{1.0 \times 10^{11}}{3-\alpha} M_{\odot} .
$$

The total amount of baryonic material (stars plus interstellar medium) in the inner Galaxy can be estimated by modeling these components, and their gravitational influence can be calculated. After conducting such a census, Olling \& Merrifield (2001) concluded that this material has the same gravitational influence as a spherical distribution of mass $M^{\text {baryon }}\left(R_{0}\right) \approx 5.7 \times 10^{10} M_{\odot} \cdot{ }^{1}$

These masses can be compared to that which is inferred dynamically by equating the centripetal acceleration of an object on a circular orbit around the Galaxy at $R_{0}$ to the acceleration induced by gravity at this radius. This value depends on the rate at which an object would have to travel to follow a circular orbit of radius $R_{0}$ around the Milky Way, $\Theta_{0} \approx 220 \mathrm{~km} \mathrm{~s}^{-1}$ (Kerr \& Lynden-Bell 1986), which gives a dynamical mass of

$$
M^{\text {total }}\left(R_{0}\right)=\frac{R_{0} \Theta_{0}^{2}}{G} \approx 9.5 \times 10^{10} M_{\odot} .
$$

\footnotetext{
${ }^{1}$ This is not quite the same as the total mass of baryonic material, since this material is not in reality distributed spherically.
} 
Subtracting $M^{\text {baryon }}\left(R_{0}\right)$ from this total mass gives a measure of the contribution from dark matter, which can be equated to the value in Equation 1. Thus, we can solve for the only unknown in Equation 1, the power law index $\alpha$, from which we find a value of $\alpha \approx 0.4$.

Binney \& Evans (2001) performed a similar but much more thorough analysis in which they looked at the enclosed mass at all radii in the galaxy (by fitting to the full rotation curve rather than just the local value of the circular velocity at $R_{0}$ ). They also used the microlensing optical depth toward the Galactic center to constrain the baryon density in the inner Galaxy. Since the microlensing measurements place a similar amount of mass in baryons, $M^{\mu \text { lens }}\left(R_{0}\right) \approx 4 \times 10^{10} M_{\odot}$, and the strongest limits on the halo mass come from the circular speed near the Solar radius in the Galaxy, they reached a similar conclusion that $\alpha \lesssim 0.4$.

There are also other lines of evidence that the dark matter distribution in the middle of the Milky Way cannot be strongly centrally concentrated. In particular, the Milky Way is known to be a barred galaxy, and the rate at which its central bar rotates tells us something about the distribution of dark matter in the vicinity. Modeling of the $\mathrm{HI}$ and $\mathrm{CO}$ kinematics near the Galactic center (Englmaier \& Gerhard 1999), associating features in the stellar distribution of the Galaxy with orbital resonances (Sevenster 1999) and Lagrange points (Binney, Gerhard \& Spergel 1997), and direct measurements of the pattern speed (Debattista, Gerhard \& Sevenster 2002) all indicate that the bar is rotating rapidly. In this context, "rapid" means that the bar pattern is rotating at such a speed that it travels at approximately the same speed at which stars near the end of the bar orbit the Galaxy. This is as fast as a bar pattern can rotate, but there is no reason why it has to travel so quickly. Indeed, if a bar is embedded in a massive dark halo, then simulations have shown that the transfer of angular momentum from the bar to the dark halo should rapidly slow the bar from this maximum speed (Debattista \& Sellwood 2000). Therefore, the rapid observed rotation speed of the Galactic bar provides evidence that the Milky Way cannot have a lot of dark matter near its center to absorb the bar's angular momentum. This argument implies that any central cusp in the dark matter distribution must be rather weak, in line with the value of $\alpha \approx 0.4$ derived above.

This low value for the central power law cusp in the Milky Way is not what the CDM simulations predict. There has been some disagreement amongst the simulators as to the exact value of $\alpha$, with a lengthy argument as to whether it lies closer to 1.0 (Navarro, Frenk \& White 1996) or 1.5 (Moore et al. 1999). However, one thing upon which all would agree is that the central cusp of a CDM halo should have a power law index much larger than the value of 0.4 that we have inferred for the Milky Way. Thus, we would appear to have dealt a killer blow to CDM: in the one place that this type of measurement can be made reliably, the observations conflict grossly with the predictions of the theory.

However, the case for the prosecution is not quite as convincing as it might at first appear. First of all, even if a galaxy initially formed with a steep power law cusp in its dark halo as CDM requires, there are mechanisms that could have erased it by the present day. In particular, as described above, the interaction between a bar and a central cusp can redistribute angular momentum rather effectively, so a strong bar could fling dark matter out from the center of a galaxy, 
rearranging any initial cusp into a less concentrated distribution. Unfortunately, once again the theorists are unable to agree on the efficacy of this mechanism: Weinberg \& Katz (2002) have shown that a strong initial bar in the Milky Way could have wiped out a central cusp, whereas Sellwood (2003) has argued that any plausible bar would lack sufficient angular momentum to do anything significant to the dark matter distribution. It is worth noting, though, that the above simple calculation on the Milky Way implies that any redistribution of mass would have to be pretty radical: since our calculation effectively measures the total mass of dark matter inside $R_{0}$ in the Milky Way, the deficit from a primordial $\alpha \approx 1$ cusp could only be explained if the initial cusp material had been flung all the way out to beyond $R_{0}$.

A second weakness in the above argument for a low value of $\alpha$ is its reliance on various Galactic constants. There are significant uncertainties in the contributions of baryonic material to the local density in the Galaxy, which translate directly into uncertainties in the amount of the total density that must be attributed to dark matter. Further, the total dynamical mass constraint depends on the adopted values for the basic Galactic constants $R_{0}$ and $\Theta_{0}$ (see Equation 2). Thus, for example, Binney \& Evans' (2001) exclusion of an $\alpha=1$ model depends largely on the fact that such a model would require the local rotation speed to be $\approx 230 \mathrm{~km} \mathrm{~s}^{-1}$, in conflict with their adopted conventional value of $\Theta_{0}=220 \mathrm{~km} \mathrm{~s}^{-1}$. Although it might be considered inappropriate to suggest such a thing at an IAU-sponsored symposium, it is likely that the IAUsanctioned "official" values of the Galactic constants are in error at this level of precision.

More detailed fits to the rotation curve face the additional problem that the shape of this curve becomes rather uncertain in the innermost parts of the Galaxy, where one might hope to be able to see the effects of a central cusp. In this region, the Milky Way's bar means that material in the disk is following strongly non-circular orbits, and it is not trivial to allow for this effect in the analysis of kinematic observations to obtain a definitive rotation curve.

These uncertainties leave plenty of "wiggle room" for those who would like to hold on to the CDM paradigm. By adopting a somewhat non-standard rotation curve in its innermost parts, and by invoking a modest redistribution of halo material out to larger radii, Klypin, Zhao \& Somerville (2002) obtained a completely satisfactory match between the observed rotation curve of the Milky Way and the predictions of a multi-component model incorporating an initial CDM cusp. Thus, this test is not by any means conclusive. Further refinements in the data and modeling might in future allow a more definitive result, but the large number of systematic errors that lurk in the analysis mean that we are still a long way from such a discriminating test.

\section{Halo Shape}

One of the systematic effects that we have thus far ignored is that the dark matter halo is unlikely to be exactly spherical. If we allow the halo to be flattened, then the simple argument from which we derived a value of $\alpha \approx 0.4$ breaks down. In particular, a halo that is flattened towards the disk will concentrate the dark matter closer to the Galactic plane, so that the average 


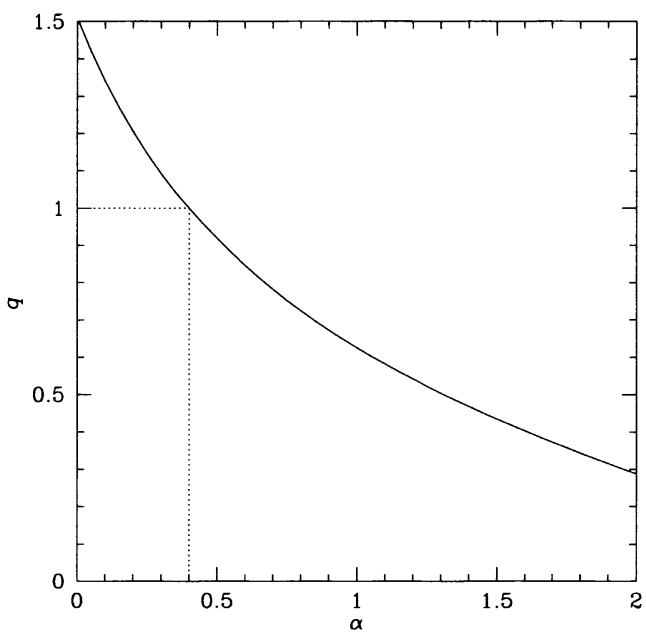

Figure 1. Plot showing the locus of a family of power law axisymmetric halos of index $\alpha$ and axis ratio $q$, all of which have the same density at unit radius in the symmetry plane. All these halos produce the same gravitational field at unit radius as a spherical $(q=1)$ halo with $\alpha=0.4$ (highlighted with a dotted line).

dark matter density at a distance $R_{0}$ from the Galactic center will be less than the value of $\rho^{\mathrm{DM}}\left(R_{0}, 0\right)$ that we measure in the Galactic plane. If we correct this over-estimate in the value that we have used to normalize the dark matter density profile, then a larger value of $\alpha$ is required to produce the same total mass of dark matter interior to $R_{0}$.

The calculation is a little more complicated than this heuristic argument would suggest, since flattening the dark matter distribution also alters its gravitational potential. Nonetheless, it is fairly straightforward to calculate the acceleration due to gravity from ellipsoidal power-law density distributions with different values of $\alpha$ and degrees of flattening as parameterized by the polarto-equatorial axis ratio $q$. One can show that all density distributions with the same value of $\rho^{\mathrm{DM}}\left(R_{0}, 0\right)$ but different values of $\alpha$ and $q$ will produce the same gravitational acceleration in the Galactic plane at the Solar radius if they have the same value for the quantity

$$
I(q, \alpha)=q \int_{0}^{1} \frac{x^{2-\alpha} \mathrm{d} x}{\sqrt{1-\left(1-q^{2}\right) x^{2}}}
$$

[c.f. Binney \& Tremaine (1987) equation (2-91)]. This integral equation can be solved by a simple iteration to obtain pairs of values of $\alpha$ and $q$ that produce the same value of $I$. Figure 1 shows the family of parameter values that produces identical values of $I$ - and hence the same acceleration in the Solar neighbourhood - as the spherical $\alpha=0.4$ halo derived in Section 2. Clearly, if we allow arbitrary flattening of the halo, we can obtain a wide range of power law indices, including the value of $\alpha \approx 1$ favoured by the simplest CDM models. 
Perhaps more interestingly, we can turn this calculation around. If it is assumed that the dark matter is distributed in an $\alpha \sim 1$ cusp, as the CDM models require, then Figure 1 shows that the halo has to be modestly flattened to an axis ratio of $q \sim 0.7$ in order to provide the correct centripetal acceleration at the Solar radius.

This value can be compared with what is found through other lines of investigation. The difficulty in measuring the shape of the Milky Way's halo by other means is that most probes of its potential only sample the equatorial plane, so provide no direct handle on the mass distribution in the polar direction. However, some tracers are affected by this third dimension.

One of the strongest tests is provided by the variation in thickness of the Galaxy's gas layer with radius. Because the amount of mass close to the plane decreases with radius, the local gravitational field in the $z$ direction also decreases. This lower field is less effective at pulling the gas to the plane, so the thickness of the layer increases with radius. However, if the dark matter halo is flattened, the dark matter is concentrated disproportionately toward the Galactic plane, increasing the vertical gravitational field and thus somewhat suppressing this tendency of the gas layer to flare with radius. Olling \& Merrifield (2000) compared the observed flaring of the Galactic gas layer to the predicted thickness of the layer in hydrostatic equilibrium in plausible mass models of the Milky Way with a variety of halo flattenings. This analysis showed that satisfactory fits could be obtained with flattenings of $0.7<q<0.9$.

An exciting new probe of the third dimension is offered by the tidal debris of merging remnants. In the Milky Way, the detritus from the Sagittarius Dwarf Galaxy has now been traced all around the sky (Majewski 2004). The fact that it forms a coherent stream over such a large angle places a strong limit on the degree of halo flattening. Only in a spherical potential would this orbit stay in a single plane; in a flattened potential, the orbit would precess around, destroying the coherence of the stream. Ibata et al. (2001) used this reasoning to argue that the carbon stars they had traced from the stream must be orbiting in a potential that is close to spherical, with $q \gtrsim 0.9$. However, the case here is still not entirely clear-cut: if the carbon stars that had been detected came from a stream that had wrapped around the Milky Way several times, as the Ibata et al. model suggested, then at any point around the stream there should be stars on different "laps" around the Galaxy, with rather different kinematics. However, the observed kinematics of these carbon stars shows a lot of coherence, suggesting that most stream members are on the same lap. If the stream is more localized in this way, then the effects of precession would be reduced, and so a flatter halo could be possible. Clearly, more data are required.

Another test of the plausibility of a flattening of $q \sim 0.7$ is by comparison with the flattenings of the halos in other galaxies. Such measurements are equally difficult in other systems, but there are a few objects with reliable measurements, based either on the gas layer flaring method, or observations of polar rings (which are similar in nature to the merger stream discussed above), or the shape of the isophotes of X-ray emission from hot gas [which provides a rather direct measure of the shape of the gravitational potential in early-type galaxies - see Buote (2004)]. The Milky Way is placed in the context of these measurements in Figure 2. The amount of data is depressingly small, but it is 


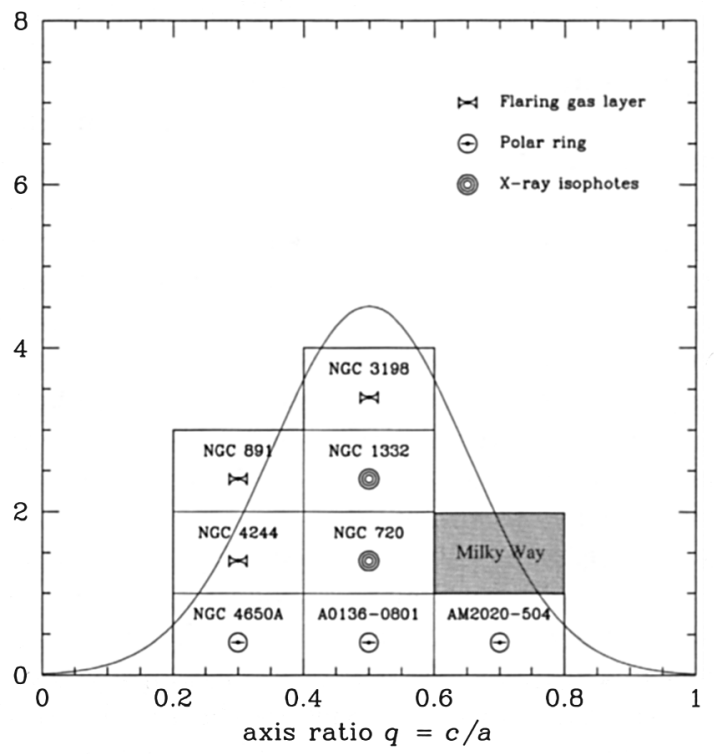

Figure 2. Histogram showing how the Milky Way fits in with the distribution of halo shapes for the handful of galaxies where reasonably reliable measurements have been made. The line shows the predicted distribution for a CDM cosmology from Dubinski (1994).

already apparent that, although the Milky Way's halo is a little rounder than average, it is by no means exceptional.

Figure 2 also shows the approximate distribution of halo flattenings that were produced in Dubinski's (1994) CDM simulations. Using this distribution as a test of the theory is rather better than looking at the steepness of any central cusp in the dark matter distribution: as discussed in Section 2, there are a number of ways of altering the primordial degree of cuspiness at the centers of halos, but the larger-scale shape of the halo is a lot harder to mutate. As is apparent from Figure 2, CDM passes this test with some ease, although the shortage of data limits the scope for detailed comparison.

\section{Conclusions}

Astronomers often proceed dangerously rapidly from saying "this theory is completely implausible" to asserting "I always said that this theory was right, and, by the way, I invented it." CDM is no exception to this overly-rapid process, so it is certainly still worth testing the theory critically wherever possible. In this regard, the unique in situ observations that one can make of the dark matter density in the Milky Way offer important tests of the theory.

Happily (or unhappily, depending on your perspective), the Milky Way seems to pass these tests fairly easily. The apparent discrepancy between the predicted density cusp of a CDM halo and the observed mass of dark matter 
in the inner Milky Way can be explained astronomically (e.g., through errors in the adopted rotation curve) or astrophysically (e.g., via redistribution of the primordial halo by a bar). One can even invoke CDM itself to explain the discrepancy: the theory generically predicts that halos should be flattened, and when such flattening is introduced in the modeling a steeper cusp is inferred for the Milky Way.

Other measures of halo shape are also more-or-less consistent with this picture. The one potentially conflicting result comes from the study of the Sagittarius Stream: if future work shows that this stream really is wrapped several times around the Galaxy without significant precession, then the Milky Way must have a halo that is very close to spherical, which would be rather uncomfortable for CDM. However, even such a strong result would not be a killer blow against CDM, since the theory predicts a wide range of halo shapes, a few of which will be close to spherical.

Indeed, this shortcoming illustrates what is probably the fundamental limiting factor in using the Milky Way as a laboratory to test CDM. The theory is sufficiently flexible that any property of the Milky Way is likely to lie somewhere within the range of possibility. The real test must surely come from comparing the properties of large samples of galaxies to the predicted distribution of the population in CDM cosmology. If, for example, star streams in other galaxies were all found to require spherical halos, then CDM would be in deep trouble.

\section{References}

Binney, J. \& Tremaine, S. 1987, Galactic Dynamics (Princeton: PUP)

Binney, J., Gerhard, O. \& Spergel, D. 1997, MNRAS, 288, 365

Binney, J.J. \& Evans, N.W. 2001, MNRAS, 327, L27

Buote, D. 2004, these proceedings

Debattista, V.P., Gerhard, O. \& Sevenster, M.N. 2002, 334, 355

Debattista, V.P. \& Sellwood, J.A. 2000, ApJ, 543, 704

Dubinski, J. 1994, ApJ, 431, 617

Englmaier, P. \& Gerhard, O.E. 1999, MNRAS, 304, 512

Ibata, R., Lewis, G.F., Irwin, M., Totten, E. \& Quinn, T. 2001, ApJ, 551, 294

Kerr, F.J. \& Lynden-Bell, D. 1986, MNRAS, 221, 1023

Klypin, A., Zhao, H.S. \& Somerville, R.S. 2002, ApJ, 573, 597

Kuijken, K. \& Gilmore, G. 1991, ApJ, 367, L9

Majewski, S.R. 2004, these proceedings

Moore, B., et al. 1999, MNRAS, 310, 1147

Navarro, J.F., Frenk, C.S. \& White, S.D.M. 1996, ApJ, 462, 563

Olling, R.P. \& Merrifield, M.R. 2000, MNRAS, 311, 361

Olling, R.P. \& Merrifield, M.R. 2001, MNRAS, 326, 164

Sellwood, J.A. 2003, ApJ, 587, 638

Sevenster, M.N. 1999, MNRAS, 310, 629

Weinberg, M.D. \& Katz, N. 2002, ApJ, 580, 627 